Revue d'histoire de l'Amérique française

सES REVUE D.HISTOIRE DE L'AMÉRIQUE FRANÇAISE

\title{
Le Séminaire de Québec et les missions d'Acadie
}

\section{Honorius Provost}

Volume 2, numéro 4, mars 1949

URI : https://id.erudit.org/iderudit/801518ar

DOI : https://doi.org/10.7202/801518ar

Aller au sommaire du numéro

Éditeur(s)

Institut d'histoire de l'Amérique française

ISSN

0035-2357 (imprimé)

1492-1383 (numérique)

Découvrir la revue

Citer ce document

Provost, H. (1949). Le Séminaire de Québec et les missions d'Acadie. Revue

d'histoire de l'Amérique française, 2(4), 613-621.

https://doi.org/10.7202/801518ar d'utilisation que vous pouvez consulter en ligne.

https://apropos.erudit.org/fr/usagers/politique-dutilisation/ 


\section{BIBLIOGRAPHIE}

\section{LE SEMINAIRE DE QUEBEC ET LES MISSIONS D'ACADIE}

Répondant à un des buts de sa fondation, le Séminaire de Québec, dès qu'il le put et jusqu'à la conquête, envoya et soutint des missionnaires en Acadie et au Mississipi. Le recrutement et la subsistance de ces missionnaires furent assurés par le concours généreux du Séminaire des Missions Etrangères de Paris, dont celui de Québec n'était qu'une succursale. Pendant près d'un siècle, il y eut ainsi un ou plusieurs prêtres en Acadie, concurremment avec des Sulpiciens, des Jésuites et même quelques Récollets; mais la direction de cette mission était dévolue aux prêtres du Séminaire. Les correspondances émanées d'eux ou échangées à leur sujet sont donc une documentation essentielle à l'histoire religieuse de toute l'ancienne Acadie. Comme ouvrages publiés spécialement sur ce sujet, nous connaissons une petite monographie rédigée en collaboration: L'Acadie, Ses missionnaires, Montréal, 1925 (48 pages), et surtout les ouvrages de l'abbé H.-R. Casgrain, notamment: Un pèlerinage au pays d'Êvangéline, Québec, 1888; Une seconde Acadie, Québec, 1894; Les Sulpiciens et les prêtres des Missions Etrangères en Acadie, Québec, 1897. L'Abbé Casgrain a également le mérite d'avoir fait publier en supplément, dans le Canada Fransais, années 1888-1890, environ six cents pages de documents inédits sur l'Acadie (1710-1815); on y trouve une petite partie de ce qui concerne les missions. Enfin l'abbé Auguste Gosselin a consacré quelques chapitres sur l'Acadie dans son ouvrage: L'Église du Canada depuis Monseigneur de Laval jusqu'à la conquête, 3 vols., Québec, 1911-1914.

Honorius Provost, ptre, sous-archiviste au Séminaire de Québec 


\section{A - DOCUMENTS CONSERVÉS AUX ARCHIVES DU SÉMINAIRE}

N.B. Las pièces énumérées ci-après, avec leurs cotes, appartiennent au vieux fonds du Séminaire. Il y aurait en outre, dans le fonds Casgrain, de nombreuses copies de documents sur l'Acadie obtenues de France ou d'Angleterre. Comme elles se trouvent également à Ottawa et peut-être ailleurs, nous ne les donnons pas ici; elles ont cependant l'avantage, chez nous, d'être cataloguées sur fiches.

1676, 5 sept. - M. Louis Petit est fait grand vicaire pour l'Acadie (Évêques, 170).

1677 - Lettre de M. Dudouyt - on a dû abandonner des églises à cause de l'eau-de-vie (Lettres N., 48 secundo, p. 5).

1678, 30 oct. - Érection de la cure de Port-Royal et collation à M. Petit (Missions I, no 3).

1681, 9 mars - M. Dudouyt se propose d'envoyer un encensoir à M. Petit (Lettres N., 52, p. 9).

1682, mars - Lettre de Dudouyt: 600" données à l'église par un domestique de Razilly (Lettres N., 61, p. 12);

1684, 10 nov. - Nouvelle érection de la cure de Port-Royal et collation à M. Petit (Missions I, no 3).

1684 - Un religieux Pénitent va à la pêche sédentaire (Lettres N., 79, p. 8).

1685, 26 avril - MM. Petit, Thury et Trouvé sont à Port-Royal (Lettres M., 1, p. 8).

1685, 13 août - Ordonnance de De Meulles défendant de porter des boissons aux sauvages de l'Acadie (Polygr. IV, no 29).

1685 - Mómoire sur les missions d'Aicadie (Missions, 8).

1687, 18 mars - Mgr de Laval trouve juste qu'on y envoie des missionnaires (Lettres N., 86).

1687, 9 juin - Lettre de Mgr de Laval: l'abbé Trouvé va en Acadie (Lettres N., 87, p. 6).

1687, 12 juin - Lettre de M. de Brisacier où il parle de M. Petit (Lettres M., 5).

1687 - Lettre de M. Dudouyt: l'abbé Trouvé va en Acadie sur l'ordre de Mgr de St-Vallier (Lettres 0, 1, p. 9).

1690 - Petit récit de la prise de l'Acadie par les Anglais (Documents F., no 167).

1692 - M. Thury doit revenir au Séminaire (Chapitre 39, p. 2).

1692 - M. Thury rencontré à Pentagoët - lettre de M. Tremblay en 1734 (Polygr. IX, 103).

1694, 3 avril - Lettre de M. Tremblay: à propos de M. Thury et de sa mission (Lettres M., 16, p. 4).

1695, 28 mai - Lettre de M. Tremblay: M. Deschambeau est à Pentagoët (Lettres M., 20, p. 80). 
1695 - Mémoire où il est question de l'abbé Thury, d'autres missionnaires (Lettres 0, 7, passim).

1696 - Notes sur la mission et M. Thury, sur M. de St-Cosme, etc. (Lettres 0,12 , passim)

1697, 3 mai - Sémin. de Paris écrit de prendre soin de M. Petit - question aussi de M. Deschambeau ('Lettres M, 24, pp. 3 et 4).

1698, 4 mai - Lettres patentes de Mgr de St-Vallier pour les missions du Séminaire en Acadie (Polygr. IX, 20).

1698, 4 mai - Lettres de gr. vicaire pour M. Thury (Polygr. IX, 21).

1698, 12 mai-M. Thury nommé supérieur de toutes les missions de l'Acadie (Polygr. IX, 23).

1698, 6 juin - Lettre du Sém. de Paris: question de M. Thury et de sa mission (Lettres M., 25, p. 4).

1698, 8 août - Érection de la cure des Mines (Polygr. IX, 22).

1698,8 oct. - M. Glandelet à Boudon: missions que l'on vient de prendre en Acadie (Sém. 6, no 73).

1698 - Le Séminaire doit y avoir des missions (Lettres 0, 23, p. 16).

1698 (circa) - Règlement et avis pour les missionnaires (Polygr. IX, 28).

1698 (avant 1699) - Lettre de Mgr de St-Vallier sur le baptême - des sauvages - copie (Sémin. 15, no 66).

1699, 1 mai - Lettre de Tremblay: question de la mort de M. Deschambeau (Lettres 0, 55, p. 17).

1699, 10 juin - Lettre du Sém. de Paris sur l'union des cures des Mines et de Pentagoët, etc. (Lettres M, 26 tertio).

1699, 1 août - Lettre de M. de Maizeret sur la mort de MM. P. Thury et Deschambeau (Lettres S, 101).

1699, 7 sept. - Lettre de M. Maudoux, à Port Royal (Missions, 89).

1699 - L'on affirme que M. Thury ne fait pas la traite (Lettres 0,55 , p. 18).

1700, 27 avril - Lettre de M. Tremblay - re: mort de M. Thury, etc. (Lettres 0, 26, pp. 17 et seq.).

1700, 26 juin - Provision de la cure des Mines pour M. Leveyer, copie (Missions, 16).

1700 - Lettre de M. Tremblay: il faut y avoir toutes les misssions ou rien (Lettres 0, 28, passim).

1701, 19 sept. - Lettre de M. Leveyer (Missions, 96).

1701, 19 sept. - M. Gaulin à M. Tremblay (Lettres R, 178).

1701, 24 oct. - Lettre de M. Gaulin à... (Lettres R, 179).

1701 - Lettre de M. Tremblay: question de M. Leveyer (Lettres N, 114, p. 27).

1701 - Lettre de M. Tremblay: on demande à Brouillan de renvoyer $M$. Guay (Lettres 0, 31, p. 27).

1702, 5 oct. - Pouvoirs accordés aux MM. Rageot et Gaulin pour l'Acadie (Polygr. IX, 23). 
1702, 23 mai - Le Sém. de Paris à celui de Québec: pas d'apparence qu'on puisse avoir un établissement solide en Acadie (Lettres M, 28).

1702, 28 nov. - M. Gaulin à M. Tremblay (Lettres R. 180).

1702 - Plusieurs communautés demandées pour l'Acadie; Pentagoët cédée aux Jésuites (Lettres N, 117, pp. 9 et 10).

1702 - Lettre de Paris: conseiller à M. Leveyer de repasser en France (Lettres 0, 36, p. 41).

1702 - Lettre de M. Tremblay: relations entre MM. Guay et Leveyer (Lettres 0, 37, p. 9).

1703, 15 juin - Les Prémontrés en Acadie; question de M. Rageot (Lettres N, 121, p. 7).

1703, 23 juin - Sém. de Paris à celui de Q.: M. Ragot envoyé en Acadie pour aider M. Gaulin (Lettres M., 29).

1703 - Lettre de M. Tremblay: il rappelle en France M. Leveyer (Lettres 0,39, p. 29).

1703 - Lettre de M. Tremblay: état de la mission d'Acadie (Lettres 0, 40, p. 65).

1704, 28 oct. - Pouvoirs accordés à M. Gaulin pour Plaisance (Polygr. IX, 23).

1706, janvier - M. Tremblay à Mgr de St-Vallier (Lettres P, 8, p. 13).

1707, 18 mars - Lettre de M. Tremblay: question de M. Gaulin (Lettres M., 35, p. 5).

1707, 8 mai - Lettre de M. Tremblay: non content de M. Gaulin (Lettres $\mathrm{M}, 37, \mathrm{pp} .4$ et 5 ).

1707, 18 juin - M. Tremblay proteste contre la conduite de M. Gaulin (Lettres M, 38, p. 11).

1710, 14 juin - Lettre de M. Tremblay - M. Gaulin a perdu un vaisseau pris par les Anglais (Lettres M, 43).

1711 - Lettre de M. Tremblay: question de M. Gaulin (Lettres 0, 51, p. 20).

1711 - Plan pour faire un établissement au Cap-Breton; lettre de $M$. Tremblay (Lettres 0,52, p. 24).

1726, 2 sept. - Érection de la cure de Louisbourg et son union au Séminaire de Q., signée par Mgr de St-Vallier (Missions, I, no. 4).

1727, 26 mars - La cure de Louisbourg ne peut convenir au Séminaire; lettre de Montigny (Lettres M, 52).

1728, 25 sept. - Lettre de M. Courtin au sujet de Louisbourg (Missions, no 7).

1728, oct. - Lettre de M. Brault, miss. à Louisbourg (Missions, no 92). 1729 - Demande de M. Gaulin à revenir au Séminaire; un canonicat pour lui ? (Lettres M, 67).

1732, 28 mai - Lettre de M. de Montigny: question de M. Courtin (Lettres $\mathrm{M}, 77)$.

1732, 2 sept. - M. de St-Poncy part pour son voyage d'Acadie (Sémin. 5 , no 57). 
1733 - Mort de M. Courtin; M. Gaulin voudrait retourner en Acadie; le Séminaire refuse (Lettres M, 82, passim).

1734, 12 sept. - Lettre de M. de St-Poncy à M. de Brisacier (Missions I, no $6 a$ ).

1734 - Mémoire: on signale la mort de M. Courtin (Sémin. 5, no. 50, p. 4). 1734 - M. de St-Vincent est envoyé à l'Ile Royale (Lettres M, 83, p. 6). 1735, 2 sept. - Louisbourg: M. Maillard à M. de Montigny (Lettres P, $60)$.

1735, 15 sept. - Lettre de M. de St-Vincent à M. de Brisacier (Missions I, no 6 b)

1735 - Comptes du Séminaire pour les missions de l'Acadie (Missions, no 105).

1736, 1 juin - Lettre de Paris: question de M. de St-Poncy (Lettres M, 88, p. 6 ).

1737, 30 avril - Lettre de Mgr Dosquet au Ministre: blâme contre M. de St-Vincent; copie (Sémin. 14, liasse V).

1737, 18 mai - Question du rappel de MM. de St-Vincent et de St-Poncy (Lettres M, 90, passim).

1737, 24 oct. - Louisbourg: lettre de M. Maillard à ...(Lettres P, 61).

1737, 28 oct. - Louisbourg: Lettre de M. Maillard à M. Collet (Lettres P, $62)$.

1738, 4 avril - Lettre de M. de Montigny: M. de St-Vincent est arrivé de l'Ile Royale (Lettres M, 91, p. 2).

1738, 15 mai - Lettre du Sémin. de Paris; retour de M. de St-Vincent et de M. de St-Poncy (Lettres M, 93, pp. 4 et 9).

1738, 29 sept. — Louisbourg: M. Maillard à ...(Lettres P, 63).

1738, 1 oct. - Louisbourg: M. Maillard à... (Lettres P, 64).

1739, 10 mai - Nous n'envoyons pas de missionnaires cette année; Lettre de Paris (Lettres M., 95, p. 3).

1739, 16 mai - Lettre de M. de Montigny: M. de St-Vincent reste en France (Lettres M, 96).

1740, 20 mai - Séminaire de Paris: question de M. de St-Poncy (Lettres $R, 8)$.

1740, 22 août - Lettre de M. de St-Poncy: il repasse en France (Lettres S, 103).

1740 (circa) - Mémoire sur les missions d'Acadie, peut-être par M. Leloutre (Polygr. IX, 29).

1741, 10 mai - Lettre de Paris: question de M. Girard (Lettres M, 99, p. 6).

1742, 27 sept. - Un testament de M. de Miniac (Sémin. III, 51).

1742, 12 oct. - Départ de M. de Miniac pour l'Acadie - note de M. Jacrau (Lettres S, 7c).

1742. 2 déc. - M. de Miniac à M. Jacrau (Lettres S, 7a). 
1743, 20 oct. - Le Gouverneur anglais fait difficulté de recevoir MM. de Miniac et Girard; lettre de Mgr de Pontbriand; copie (Sémin., 14, liasse VI).

1743 - M. Miniac à M. Jacrau (Lettres S, 7e).

1744, 26 avril - M. Miniac à M. Jacrau (Lettres $S, 7 \mathrm{~g}$ ).

1744, 3 mai - M. Miniac à M. Jacrau (Lettres S, 7i).

1744, 17 juin - M. de Bourgerie à M. Miniac (Polygr. VII, 116).

1744, 2 oct. - M. Miniac à M. Jacrau (Polygr. VII, 115).

1745, 22 sept. - M. Miniac à M. Jacrau (Polygr. VII, 113).

1745, 23 sept. - Lettre de M. de Miniac à ...(Lettres T, 57).

1745, 1 déc. - Lettre de M. de Miniac à M. Vallier (Lettres T, 59).

1746, 10 nov. - Lettre de Mgr de Pontbriand au Ministre, défendant les missionnaires - copie (Ś́min. 14, liasse VI, pp. 12 et 13).

1747, 20 avril - M. Miniac à M. Jacrau (Polygr. VII, 117).

1747, 10 juillet - Lettre de Mgr de Pontbriand: M. Miniac doit revenir de Beaubassin - copie (Sémin. 14, liasse VI).

1747, août - Lettre de M. de Miniac à ...(Polygr. VII, 111).

1747, 14 oct. - Lettre de M. de Miniac à ...(Polygr. VII, 110).

$1747-M$. Girard demande privilèges pour l'église de Cobequid (Lettres R. 90.).

1747 - Aventures de l'abbé Leloutre fait prisonnier (Lettres R, 91).

1747 - M. Miniac en comptes avec M. Jacrau (Polygr. VII, 119).

1748, 4 juin - M. de Miniac à M. Jacrau: il va à la mission de M. Girard (Polygr. VII, 109).

1749; 11 fév. - Lettre de M. de Miniac sur les missions d'Acadie (Polygr. VII, 107).

1749, 14 avril - Lettre de M. de Miniac à M. Jacrau: sur son départ de Louisbourg (Polygr. VII, 106).

1749, 8 sept. - Testament de M. de Miniac à Louisbourg et deux lettres du même (Poblygr. VII, 121 et 122; Lettres $S, 7 j$ ).

1749, 18 oct. - Lettre de M. Maillard, de Louisbourg (Lettres P, 66). 1749 - On paye pour l'embarquement de M. Leloutre à Larochelle (Sém. 8 , no 36$)$.

1749 (circa) - Lettre de M. de Miniac à M. Jacrau (Polygr. VII, 108).

1750, 7 oct. - Lettre de M. Maillard, de Louisbourg (Lettres P, 67).

1751, 8 juin - Lettres de Paris: question des abbés Leloutre, Manach, etc. (Lettres M, 115).

1751, 10 oct. - M. Manach à M. Burguerieux (Missions, 95).

1751, 13 oct. - M. Maillard à ..., de Louisbourg (Lettres P, 68).

1751, 29 oct. - M. Manach à..., de Beauséjour (Missions 97).

1752, 5 avril - M. Manach à M. Dufau (Missions 94).

1752, 21 juin - Lettre de M. Maillard, de Louisbourg (Lettres P, 69). 1754 - Le Séminaire supporte les missionnaires (Sémin. 5, no 53).

1756 (circa) - M. Maillard à Mme Drucourt au sujet des Micmacs (Lettres R, 190). 
1757, 24 août - Vaudreuil à M. LeGuerne: ne peut secourir les Acadiens (Lettres P, 83).

1757, 30 oct. - Observation de Mgr de Pontbriand -- copie (Sémin. 14, liasse VI).

1758, août - M. Maillard écrit au sujet de Boishébert au siège de Louisbourg (Lettres $P, 70)$.

1758 - Liste des missionnaires d'Acadie avant la prise de Louisbourg (Polygr. VII, 5).

1759, 16 fév. - Lettre de Paris: M. Girard a été conduit en France (Lettres $\mathrm{M}, 117)$.

1760, 14 f'év. - Lettre de Paris: M. Leloutre toujours prisonnier (Lettres $\mathrm{M}, 118)$.

1760 - Lettre de M. Maillard à..., de Louisbourg (Lettres P, 71).

1760 (ou après) - Lettre de M. Maillard (?) à un officier anglais (Lettres $\mathrm{P}, 72$ ).

1762, 28 fév. - Séminaire de Paris à celui de Québec: on veut enlever M. Maillard aux missions (Missions, 99).

1763, 11 sept. - Libération à cette date de M. Leloutre - annoncée dans une lettre de Paris du 31 mars 1764 (Lettres M, 121).

1763-1764 - Mémoires de réclamations de MM. Leloutre, Girard, etc. contre le Sém. de Paris - imprimés (Polygr. XI, 2, 3, 4).

1764, 10 avril - Lettre de Paris: question de M. Girard (Lettres M, 122).

1764, 6 sept. - Arrêt du Parlement re: M. Leloutre (Polygr. V, 40).

1764, 6 sept. - Notes sur les missions de l'Ile Royale (Missions I, no 1).

1764, 6 sept. - Mémoire pour l'Acadie, par M. de la Goudalie (Missions, no 9).

1764, 6 sept. - Mémoire: les Sœurs de Louisbourg sont trèspauvres (Polygr. VII, nos 10 et 41 ).

\section{B - DOCUMENTS CONSERVÉS AUX ARCHIVES DE L'ARCHEVÊCHÊ DE QUÉBEC}

N.B. Le Rapport de l'Archiviste de la Province de Québec, années 1939-40,1940-41 et 1941-4.Q, a publié un inventaire des documents pour l'histoire de l'Église du Canada sous le régime français, préparé par feu l'abbé Ivanhoë Caron; les documents concernant les missions d'Acadie consarvés aux archives de l'Archevêché de Québec y ont été inventoriés, mais seulement jusqu'à l'année 1729, date où la mort de l'auteur a suspendu son travail; la liste ci-auprès servira de supplément.

1731, 8 sept. - Nécessité de mettre un grand vicaire à Louisbourg (Egl. Can., I, 99).

1736, fǿvrier - Articles concernant la construction d'une église à Louisbourg (Egl. Can., I, 257, 258).

1740, 31 août - Lettres de grand vicaire à l'abbé A.-S. Maillard (Reg. B, f. 324 ro). 
1740,21 oct. - M. de St-Poncy est fait grand vicaire (Reg. B, f. 333 vo). 1742, 20 août - Mandement aux missionnaires concernant la conduite de leurs paroisses (Reg. C., f. 170 ro).

1743, 20 oct. - Le gouvernement anglais a fait difficulté pour recevoir les missionnaires; lettre de Mgr de Pontbriand (Lettres II, 513).

1744, 29 oct. - Mandement d'actions de grâces pour les victoires du Roi de France et la prospérité de la colonie (Reg. C., f. 185 ro).

1746, 14 mars - Versailles: les missionnaires que les Anglais ont laissés en Acadie recevront leur pension comme auparavant (F., I, 92).

1746, 10 nov. - Lettre de Mgr de Pontbriand: que les missionnaires se conduisent de façon à pouvoir se maintenir (L'ettres II, 523).

1748, 6 sept. - Mgr de Pontbriand tâchera d'envoyer deux missionnaires (Lettres II, 681).

1748, 7 nov. - Mgr de Pontbriand croit devoir remplacer les Récollets par des séculiers à Louisboưrg (Lettres II, 686).

1749 - Lettre de France: Les Récollets et les Frères de la Charité reviennent (F I, 102).

1750, 4 avril - Paris: Sur les limites de l'Acadie. Conduite des Anglais envers les Acadiens. État des missionnaires (V.G., III, 51).

1750 - Lettre de France: Les missionnaires doivent être circonspects avec les Anglais (F I, 104).

1750 (circa) - Paris: Moyens proposés par l'abbé de l'Isle Dieu pour affermir

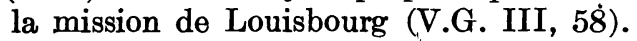

1751, 23 mai-Paris: Prétentions des Anglais. La Cour s'occupe des habitants persécutés (V.G. III, 68).

1752, 28 mars - Paris: On demande à la Cour des missionnaires pour aider l'abbé Leloutre (V.G. III, 81).

1752, 17 avril - Paris: Démarches par l'abbé de l'Isle Dieu pour procurer des missionnaires (V.G. III, 101).

1752, 19 mai - Paris: Triste situation des habitants de l'Acadie (G. III, 106).

1752, 24 juillet - Paris: Départ de deux miss. pour les Acadiens; il faut éloigner ceux-ci de la domination des Anglais (V.G., III, 133).

1752, 16 août-Pouvoirs extraordinaires accordés au Père Fournier (Reg. C, f. 215 ro).

1753, 14 janv. - Pouvoirs extraordinaires accordés à l'abbé C.J.B. Chauvreux (Reg. C, f. 216 ro).

1753, 30 janv. - Paris: Plan de cantonnement proposé pour les Acadiens (V.G. III, 152, 156).

1753, 27 fév. - Pouvoirs extraordinaires accordés à l'abbé Le Guerne (Reg. C, f. 218 ro).

1753, 16 mars - Paris: Encouragement aux Acadiens. Deux nouveaux missionnaires (V.G. III, 175, 178).

1753, 1 avril - Paris: Pensions aux missionnaires sous la juridiction anglaise (V.G. III, 186). 
1753, 1 avril - Tableau exact de l'Acadie et de ses limites dressé par MM. de l'Isle Dieu et Leloutre (V.G. III, 189).

1753, 9 juillet - Paris: La mission de Louisbourg ne progressera pas tant qu'elle sera aux Récollets (V.G. IV, 25).

1754, 20 mars - Lettres de grand vicaire à M. Leloutre (Reg. C, f.217vo).

1754, 29 mars - Paris: Renseignements sur les missions et missionnaires (V.G. IV, 58).

1754, 1 avril - Paris: Il faudrait envoyer quatre missionnaires à l'Acadie française et deux à l'Acadie anglaise (V.G. IV, 63).

1754, 10 mai - Paris: Expédition d'une caisse dé livres et d'ornements d'église à Louisbourg (V.G. IV, 75).

1754, mai-juillet - Paris: plusieurs lettres où l'on parle des missionnaires retardés à leur départ pour l'Acadie (V.G. 83 à 97).

1754, 5 sept. - Lettres de grand vicaire à M. Maillard et instructions pour la desserte de ses missions (Reg. C, ff. 220 et 221).

1755, 15 janv. - Confirmation de concession d'un terrain à l'Ordre de Saint-Jean de Dieu (Égl. Can. I, 224).

1755, mars-mai - Paris: mémoires sur les missions et missionnaires (V.G. IV, 128, 130, 136, 137, 141).

1755, 6 oct. - Lettres de grand vicaire à l'abbé Girard pour l'Isle St́́Jean (Reg. C, f.222ro).

1755, 8 oct. - Les trois missionnaires destinés à Louisbourg se sont rendus à Québec (V.G. IV, 144).

1756, 28 mars - Préparatifs des Anglais; postes de missions, etc. (V.G. IV, 153, 156).

1756, 22 juin - Nouvelles de l'Acadie: habitants pillés, massacrés, etc. (N.E., 1, 2).

1766, 18 août - Les Acadiens demandent des mîssionnaires (Égl. Can, VII, 341).

1766, 17 déc. - Rome: La Propagande dromande à l'abbé de l'Isle Dieu d'envoyer des missionnaires (Cartable C.M.R. III, 19).

1787, 17 déc. - Paris: Ce serait au Séminaire de Québec à nommer le supérieur de la mission d'Acadie - copie (V.G. VI, 27, 61). 